\title{
Understanding the structure-property relationships of lead-free piezoelectric systems
}

\author{
A. Manjon-Sanz ${ }^{1,2^{\star}}$, C. Berger ${ }^{2}$, C. M. Culbertson ${ }^{2}$, M. R. Dolgos ${ }^{2,3}$ \\ ${ }^{1}$ Neutron Scattering Division, Oak Ridge National Laboratory, Oak Ridge, TN 37831, USA \\ ${ }^{2}$ Department of Chemistry, Oregon State University, Corvallis, OR 97331USA \\ ${ }^{3}$ Department of Chemistry, University of Calgary, Calgary, Alberta, T2N 1N4, Canada \\ manjonsanzam@ornl.gov
}

Lead zirconate titanate, $\mathrm{PbZr}_{1-\mathrm{x}} \mathrm{Ti}_{\mathrm{x}} \mathrm{O}_{3}(\mathrm{PZT})$, is a ceramic perovskite material that has exceptional striking piezoelectric properties at the morphotropic phase boundary (MPB) showing a piezoelectric response $\left(d_{33}\right)$ of $200-600 \mathrm{pC} / \mathrm{N}$ [1]. This compound plays an important role in industry and has many commercial applications [2-3]. However, the toxicity of lead has spurred considerable interest in the discovery of $\mathrm{Pb}$-free ferroelectric materials. Here, we present our results on two different $\mathrm{Pb}$-free piezoelectric systems: 1) solid solutions between $\mathrm{BaTiO}_{3}(\mathrm{BT})$ and $\mathrm{BiInO}_{3}(\mathrm{BI})$, and 2) $\mathrm{BaZr}_{0.2} \mathrm{Ti}_{0.8} \mathrm{O}_{3}(\mathrm{BZT})$ and $\mathrm{Ba}_{0.7} \mathrm{Ca}_{0.3} \mathrm{TiO}_{3}(\mathrm{BCT})$.

1) Solid solutions between $\mathrm{BaTiO}_{3}$ and $\mathrm{BiInO}_{3}$ : Bismuth based perovskites are established as good ferroelectric materials, but it is still necessary to improve the piezoelectric properties of Bi-based perovskites to compete with the exceptional ferroelectric properties of PZT. To fully understand the reason of these maximized physical properties at the MPB, it is crucial to study the structure in detail. For the system $(1-x) \mathrm{BT}-(x) \mathrm{BI}$, we study the electromechanical properties and the structure of the solid solution between the Bi-based material BI with orthorhombic space group $P n a 2_{1}$ and the classical piezoelectric material BT with tetragonal structure $P 4 \mathrm{~mm}$, in the region $0.03 \leq x \leq 0.12$. Based on a structural analysis study previously carried out by Datta et al. [4], it was predicted that there is an MPB created by a polarization extension mechanism for the system at $x=0.1$. In our work, based on Rietveld analysis performed on neutron and synchrotron radiation X-ray diffraction data, we have found that a gradual structural phase transition takes place from a polar tetragonal structure $(P 4 \mathrm{~mm})$ and passes through two regions of coexisting phases: 1) $P 4 \mathrm{~mm}+R 3 \mathrm{~m}$ in the range $0.03 \leq x \leq 0.075$, and 2) $P m \overline{3} m+R 3 m$ for $0.10 \leq x \leq 0.12$. The properties also transition from ferroelectric $(x \leq 0.03)$ to relaxor ferroelectric $(x \geq 0.05)$ as the dielectric permittivity maximum becomes temperature and frequency dependent. This transition was also confirmed via polarization-electric field measurements as well as strain-electric field measurements. At the critical composition of $x=0.065$, a moderate strain of $\sim 0.104 \%$, and an effective piezoelectric coefficient $\left(d_{33} *\right)$ of $260 \mathrm{pm} / \mathrm{V}$ were observed. The original purpose of this study was to demonstrate the polarization extension mechanism as predicted in the literature, but due to the ferroelectric to relaxor transition, this mechanism was not found to be present in this system. However, this demonstrates that $\mathrm{BaTiO}_{3}$-based lead-free ceramics could be modified to obtain enhanced electromechanical properties for actuator applications [5].

2) Solid solutions between $\mathrm{BaZr}_{0.2} \underline{\mathrm{Ti}}_{0.8} \underline{\mathrm{O}}_{3}$ and $\mathrm{Ba}_{0.7} \underline{\mathrm{Ca}}_{0.3} \underline{\mathrm{TiO}}_{3}$ : The solid solution $(1-x) \mathrm{BZT}-x \mathrm{BCT}$ is the first $\mathrm{Pb}$-free piezoelectric material with a significantly high enough $d_{33} \sim 620 \mathrm{p} C / \mathrm{N}$ at the MPB at $x=0.50$, that has the potential to replace the industry standard PZT in certain applications [6]. So far, lots of studies have focused mainly in investigating the physical properties. However, the two structural characterization works of the structure at the MPB for BZT-xBCT, using solely synchrotron X-ray diffraction data, yield different results [7-8]. Here, we re-investigate the phase diagram of $(1-x)$ BZT- $x$ BCT as a function of temperature using high quality neutron powder diffraction data collected at POWGEN at the Spallation Neutron Source and applying the Rietveld method. We study the composition $x=0.50$ at the MPB, one composition in the rhombohedral range $(x=0.40)$, and another composition in the tetragonal range $(x=0.60)$. Neutron diffraction is a powerful tool to have more accurate information about the light elements such as oxygens. So, this work is crucial to investigate the octahedral tilts of $(1-x)$ BZT-xBCT materials, and further understand how the structure has an impact on their physical properties. We expect to obtain a detailed description of the structures at different temperatures, solve the debate of the symmetry at the MPB, and build a phase diagram.

[1] Damjanovic, D., Klein, N., Li, J., Porokhonskyy, V. (2010) Funct. Mater. Lett. 3 (1):5-13.

[2] Panda, K. P. (2009) J. Mater. Sci. 44 (19):5049-5062.

[3] Roedel, J., Jo, W., Seifert, K. T. P., Anton, E. M., Granzow, T., Damjanovic, D. (2009) J. Am. Ceram. Soc. 92 (6):1153-1177

[4] Datta, K., Suard, E., Thomas, P. A. (2010) Appl. Phys. Lett. 96 (22):221902-221903.

[5] Manjon-Sanz, A., Berger, C., Dolgos, M. R., J. Mater. Sci. (2017) 52:5309-5323.

[6] Liu, W., Ren, X. B., Phys. Rev. Lett., (2009) 103, 257602.

[7] Keeble, D. S., Benabdallah, F., Thomas, P. A., Maglione, M., Kreisel, J. (2013) Appl. Phys. Lett., $102(9) 092903$.

[8] Haugen, A., Forrester, J. S., Damjanovic, D., Li, B., Bowman, K. J., Jones, J. L, (2013) J. Appl. Phys., $113,014103$.

Keywords: Lead-free piezoelectrics, morphotropic phase boundary, structural analysis. 